\title{
Millimeter Wave Bandpass Filter Design Based on Support Vector Machine
}

\author{
Sun $\mathrm{Lu}^{1}$, Hu Huifeng ${ }^{2}$, Hu Cong ${ }^{3}$ \\ ${ }^{1,2}$ School of Mechano-Electronic Engineering \\ Xidian University \\ XI'AN, Shaanxi, P.R.CHINA \\ ${ }^{3}$ School of Electronic Engineering and Automation \\ Guilin University of Electronic Technology \\ Guilin, GuangXi, P.R.CHINA
}

\begin{abstract}
In the design of millimeter wave $E$ plane waveguide filter, E-Plane septum modeling and analysis is a key link. Accuracy and speed of modeling method will greatly affect the design of filter. It is proposed to use support vector machine (SVM) to the accurate modeling of E-Plane septum. Compared with the previous numerical analysis method, this method has obtained the faster speed and better accuracy. Using the modeling method, the speed and accuracy of millimeter wave $E$ plane waveguide filter can be improved.
\end{abstract}

Keywords_E-Plane septum; Support Vector Machine; Modeling speed; Millimeter wave filter

\section{INTRODUCTION}

Among millimeter wave filters, E-Plane septum waveguide filter, because of its simple structure, easy fabrication and installation, can obtain high Q value and the advantage of good filter characteristics. It has a wide range of applications. In the design of this filter, the analysis of the E plane septum is very important.

There are many electromagnetic field numerical analysis methods before such as the residue analysis method, the finite element method, the pattern matching method and FDTD (finite difference time domain) method which can compute parameters of these E-plane septum .These numerical analysis methods have high precision, but they will cost longer time.

In the different analysis and design software of filters, it is required to call the analysis program of E-Plane septum repeatedly, the speed and accuracy of the program is of prime importance to the design of filter.

In this paper, SVM is introduced to model the

This paper is supported by the National Natural Science Foundation of China (Grant No. 61501344, No.61404097) and the open foundation of Guangxi Automatic detection and Instrument Laboratory (Grant No. YQ15205) relationship between sizes of E plane septum and parameters of equivalent circuit. After replacing traditional electromagnetic field numerical analysis methods with SVM, the speed and accuracy of filter design can be improved well.

\section{II . EQUIVALENT CIRCUIT OF E PLANE METAL SEPTUM WITH PATTERN MATCHING METHOD}

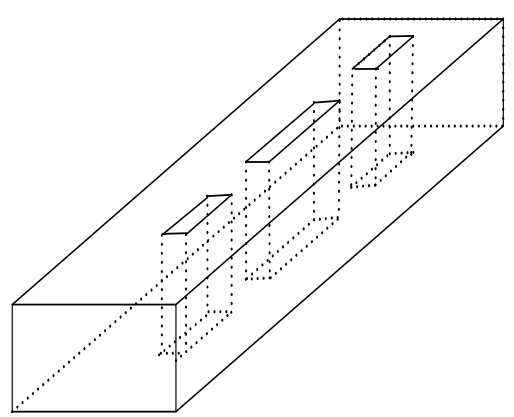

Fig. 1. Three-dimensional structure of E plane insert filter

Structure of rectangular waveguide $\mathrm{E}$ mask filter is shown in figure 1 ,two or more metal septums ${ }^{[1]}$ are inserted in the middle of the E plane rectangular waveguide and they are parallelled with each other. They have a certain distance between themselves with coupling effect and provide a resonant cavity .Using method of pattern matching ${ }^{[2-3]}$, S parameters of E plane septum can be calculated. And using the method of network cascade, parameters $\mathrm{S}$ of the total waveguide section and the septum can be got. From these results, we can get the optimized size of the filter.

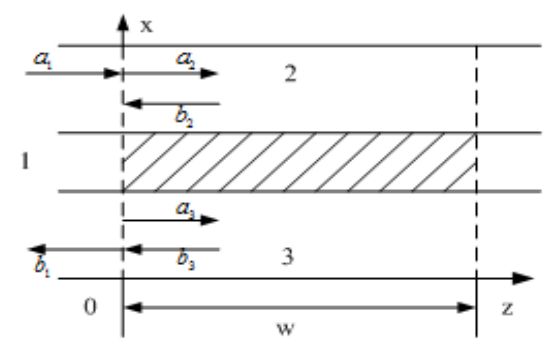

Fig .2.Basic unit of the filter 


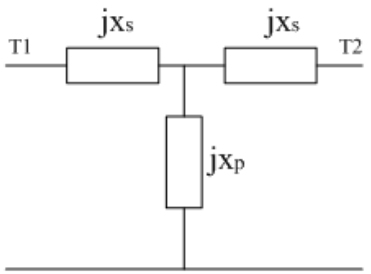

Fig. 3. Equivalent circuit of E plane septum

Structure of filter is composed of a series of basic units as shown in figure 2.In figure 2, section 2 and section 3 can be regarded as two small rectangular waveguide, $\mathrm{z}=0$ and $\mathrm{z}=$ $\mathrm{w}$ as two of the rectangular waveguide discontinuity. They can be analyzed with pattern matching method. After the numeric computation, E-plane septum ${ }^{[4]}$ can be described as $\mathrm{T}$ equivalent circuit in fig 3.

$$
\begin{gathered}
\mathrm{j} X_{s}=\frac{1-s_{12}+s_{11}}{1-s_{11}+s_{12}} \\
j X_{p}=\frac{2 s_{12}}{\left(1-\mathrm{s}_{11}\right)^{2}-s_{12}{ }^{2}}
\end{gathered}
$$

Then the impedance converter $\mathrm{K}$ can be calculated as:

$$
\begin{gathered}
\phi=-\tan ^{-1}\left(2 X_{p}+X_{s}\right)-\tan ^{-1} X_{s} \\
K=\mid \tan \left(\phi / 2+\tan ^{-1} X_{s}\right)
\end{gathered}
$$

With the impedance converter $\mathrm{K}$, we can accomplish the design of the $\mathrm{E}$ plane filter. In the traditional design of $\mathrm{E}$ plane filter, if the rectangular waveguide, the thickness and position of septum, the frequency have been selected, we can get the parameter $\mathrm{S}$ with the variable of length w. Some results of the computation can be seen in table 1.But in this computation, it will cost a long time with the method of pattern matching method. So in this paper, we want to adopt the method of support vector machine ${ }^{[5-7]}$ to replace the traditional method to reduce the computation time.

TABLE I. Transformation from the symmetric diaphragm parameters to the equivalent circuit parameter

\begin{tabular}{|c|c|c|c|c|c|c|}
\hline & \multicolumn{2}{|c|}{$33 \mathrm{GHz}$} & \multicolumn{2}{c|}{$35 \mathrm{GHz}$} & \multicolumn{2}{c|}{$37 \mathrm{GHz}$} \\
\hline $\mathrm{W}(\mathrm{mm})$ & $x_{p}$ & $X_{s}$ & $X_{p}$ & $X_{s}$ & $X_{p}$ & $X_{s}$ \\
\hline 1 & 0.3192 & 0.1942 & 0.3811 & 0.2147 & 0.4552 & 0.2348 \\
\hline 2 & 0.1681 & 0.3082 & 0.2124 & 0.3441 & 0.2713 & 0.3804 \\
\hline 3 & 0.0919 & 0.3749 & 0.1225 & 0.4229 & 0.1668 & 0.4732 \\
\hline 4 & 0.0507 & 0.4133 & 0.0713 & 0.4706 & 0.1033 & 0.5324 \\
\hline
\end{tabular}

BJ320 （ $\mathrm{a}=7.112 \mathrm{~mm}, \mathrm{~b}=3.556 \mathrm{~mm} ）, \mathrm{t}=0.2 \mathrm{~mm}, \quad \mathrm{a} 1=(\mathrm{a}-\mathrm{t}) / 2, \mathrm{a} 2=\mathrm{a} 1+\mathrm{t}, 15$ patterns

\section{MoDELING METHOD OF SVM}

SVM is a creative machine learning method based on the theory of VC, the basic idea is through nonlinear transform defined by inner multiplication function, the input space transformation to a higher dimensional space. In the high dimensional space for input and output variables, a nonlinear relationship between its basic structure as shown in figure 4 . SVM has strict theoretical basis, the use of structural risk minimization principle, have good generalization ability. SVM algorithm is a convex quadratic optimization problem and the solution is global optimal solution which can well solve the small sample, nonlinear and high dimension and local minimum point.

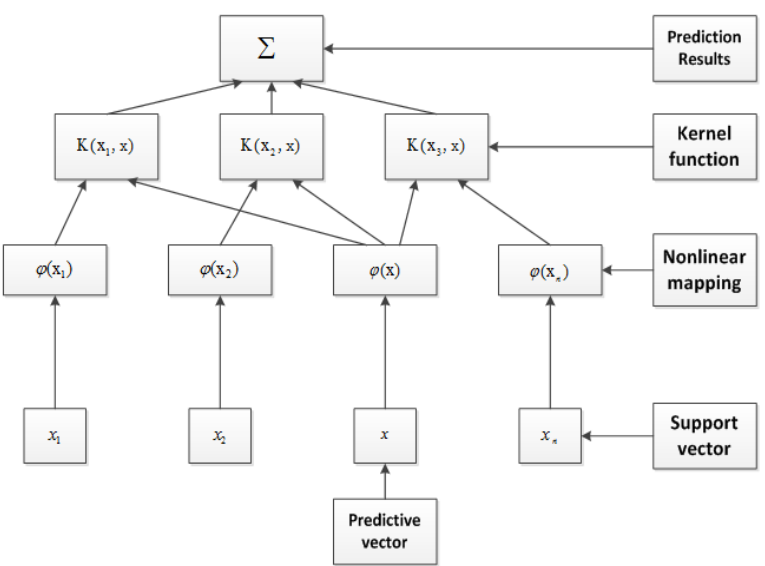

Fig. 4. Basic structure of SVM

Several groups of frequency and length of septum w are calculated using the method of pattern matching method which can be used as input samples. First, all the sample data are normalized to $[1,1]$ and the final forecast modeling results and the unitary processing. Main advantages of normalization is the effective limit data range and improve the efficiency of modeling, but also it can avoid a large amount of difficult calculation. In this paper, the $\varepsilon$ SVM is chosen to complete the function fitting.

As for waveguide BJ320, the three sample frequency $\mathrm{f}=33.0 \mathrm{GHz}, 35.0 \mathrm{GHz}$ and $37.0 \mathrm{GHz}$, to it can be calculated that the length of diaphragm and $\mathrm{T}$ - type equivalent circuit parameter value as output sample set with the pattern matching method. Data of $35.0 \mathrm{GHz}$ and 37.0 $\mathrm{GHz}$ are used as a sample point to establish the SVM model, data of $33.0 \mathrm{GHz}$ are used as network simulation to validate error. It can be seen that the SVM model established can predict the $33.0 \mathrm{GHz}$ frequency equivalent circuit parameters very well, the modeling result is shown in figure5.And the modeling procedure costs about $0.5 \mathrm{~s}$. 


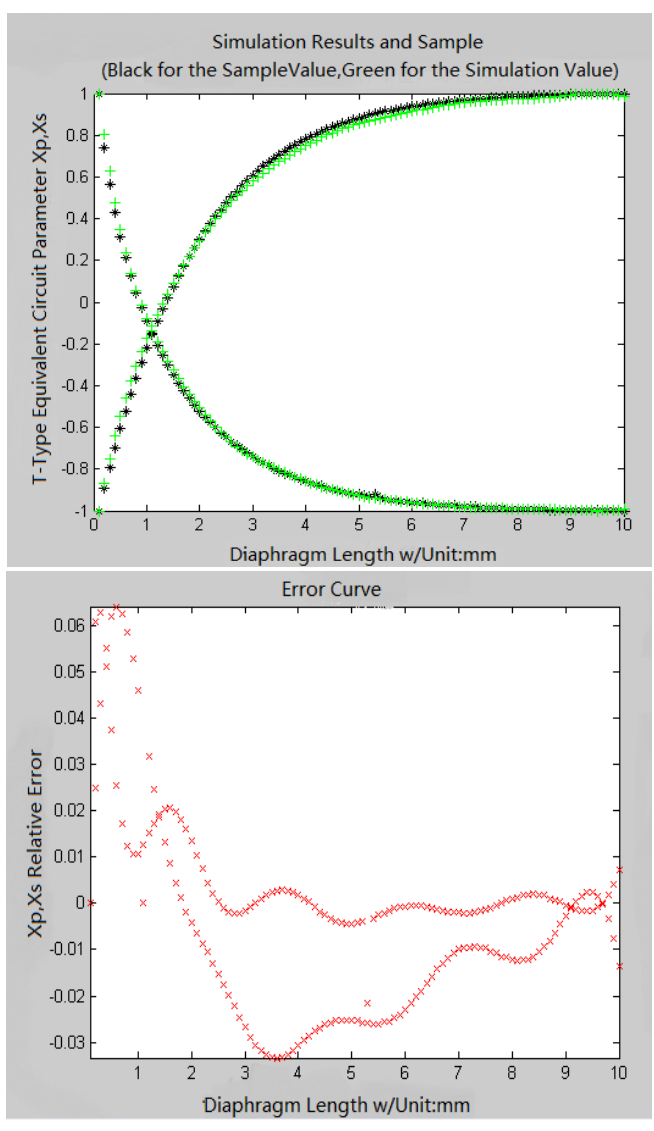

Fig.5. Modeling results of SVM

\section{IVDESIGN OF E PLANE FILTER WITH SVM MODELING RESULTS}

With the modeling method above, we can design the $\mathrm{E}$ plane filter with the procedure of fig 6.And the simulation of 34GHz band-pass filter with HFSS can be seen as fig 7.The filter has a good pass-band and cut-off characteristic.

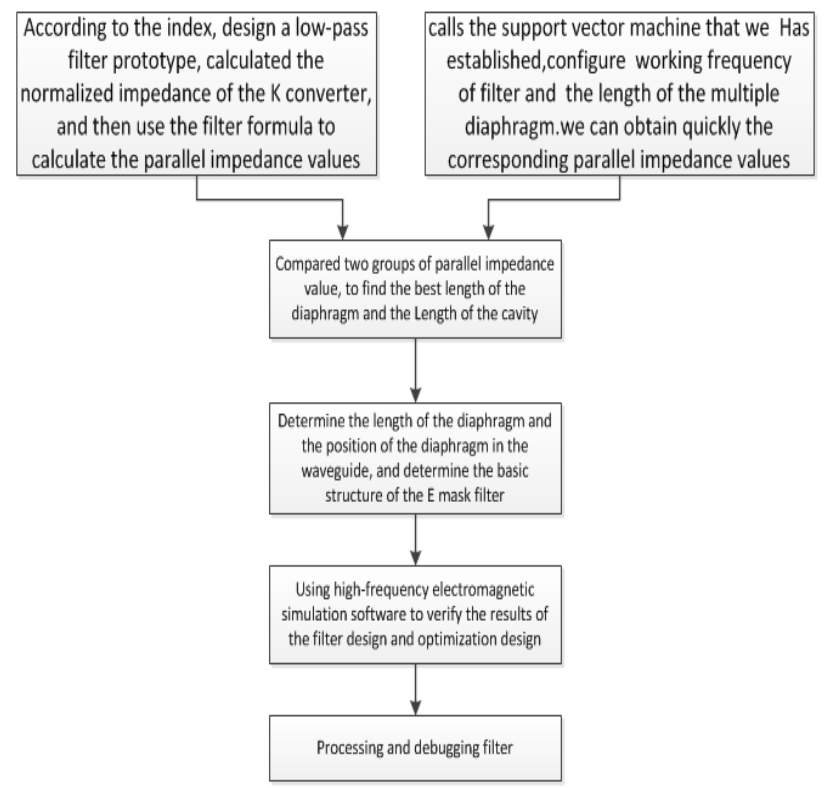

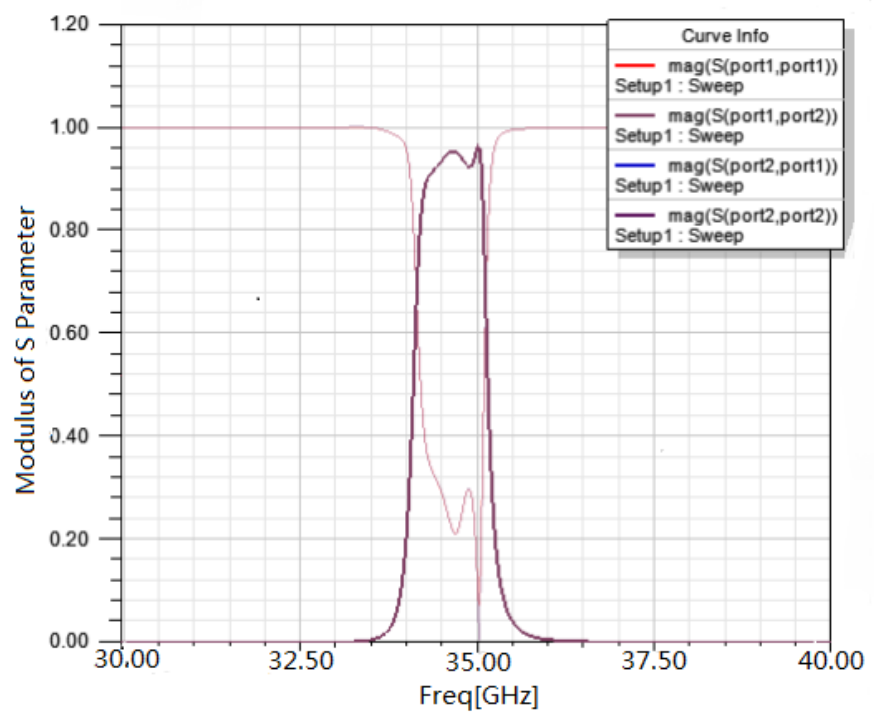

Fig 7. HFSS Simulation results of $\mathrm{E}$ plane band pass filter

\section{V.CONCLUSIONS}

It is introduced the SVM modeling method to replace the traditional electromagnetic field numerical analysis methods for the equivalent circuit model of $E$ plane septum. The speed and accuracy in the design of $E$ plane waveguide filter can be improved. This method can be a good supplement to the design of $\mathrm{E}$ plane waveguide filter.

\section{REFERENCES}

[1] TANG Hong-Jun, HONGWei, Novelm Millmeter Wave Substrate Integrated Waveguide Filter With CompactConfiguration[J], Journal of Infrared and Millimeter Waves,2006, 5(2) :139-142.

[2] SHENKai, WANGGuang-ming, QILi-hui, Design of Double Septum Waveguide Filter Based on Mode Matching Method[J], Journal of China Academy of Electronics and Information Technology, 2008,3(6):653-656.

[3] Shen K, Wang G M, Fu S H， Gu G D. Highly selective bandpass filter based on substrate integrated waveguide $[\mathrm{J}]$. Electronics Letters， 2009， 45(14): 746-748

[4] HOU Yan-ru, DING Zhi-zhong, LIU Chao, Compact Design of a Millimeter Waveguide Filter, Journal of Microwaves,2012,28(6):93-96

[5] WU Wen ,LI Xing-guo ,JIANG Jin-shui, Research on the Application of the Neural Network to the Design Method of the Millimeter Waves E-Plane Filter, ACTA ELECTRONICA SINICA, 2000，28（6）: 76-78.

[6]SU Jinshan,XIA Yierma, Filter Design Based on Least Squares Support Vector Machine (SVM), Journal of Yili Normal University ( Natural Science Edition ) ,2009,3:18-21.

[7]Q.J.Zhang,Kuldip C.Gupta,Vigay K.Devabhaktuni.Artificial Neural Networks for RF and Microwave Design-from Theory to Pratice.IEEE Tran. on Microwave Theory to Pratice.2003,51(4).1339-1350

Fig.6. Design procedure of E plane pilter with modeling results 